Mathematical Modelling and Analysis

Volume 16 Number 2, June 2011, 315-325

Doi:10.3846/13926292.2011.580788

(C) Vilnius Gediminas Technical University, 2011
www.tandf.co.uk/journals/TMMA

Publisher: Taylor\&Francis and VGTU

Online ISSN: $1648-3510$

Print ISSN: 1392-6292

\title{
Exact Periodic Wave Solutions of a Singular Integrable Equation
}

\author{
Shaolong $\mathrm{Xie}^{a}$ and Bin $\mathrm{Gao}^{b}$ \\ ${ }^{a}$ Department of Mathematics and Statistics, Business School, \\ Yuxi Normal University \\ ${ }^{b}$ Department of Physics, Faculty of Sciences, Yuxi Normal University \\ Yuxi, 653100 Yunnan, China \\ E-mail(corresp.): xieshlong@163.com \\ E-mail: gobridge@163.com
}

Received December 04, 2010; revised March 14, 2011; published online May 1, 2011

\begin{abstract}
In this paper, theory of dynamical systems is employed to investigate periodic waves of a singular integrable equation. These periodic waves contain smooth periodic waves, periodic cusp waves and periodic cusp loop waves. Under fixed parameter conditions, their exact parametric expressions are given.
\end{abstract}

Keywords: Singular integrable equation, closed orbit, smooth periodic wave, periodic cusp wave, periodic cusp loop wave.

AMS Subject Classification: 35Q51; 35Q58; 37K50.

\section{Introduction and Main Results}

Qiao [4] presented a completely integrable water wave equation:

$$
u_{t}-u_{x x t}+3 u^{2} u_{x}-u_{x}^{3}=\left(4 u-2 u_{x x}\right) u_{x} u_{x x}+\left(u^{2}-u_{x}^{2}\right) u_{x x x}
$$

where $u$ is the fluid velocity and subscripts denote the partial derivatives. This equation can be derived from the two-dimensional Euler equation by using the approximation procedure. He has proved that Eq. (1.1) has Lax pair and biHamiltonian structures, which implies the integrability of the equation. Qiao $[4,5]$ obtained the new cuspons, one-peak solitons, W-shape-peaks and Mshape-peaks solutions. Apparently, if $u(x, t)$ is a solution of Eq. (1.1), then $-u(x, t)$ is a solution also. So, when $u(x, t)$ is a W-shape-peak solution of Eq. (1.1), then $-u(x, t)$ is an M-shape-peak solution. Taking special wave speed and using integral method, Qiao [4] showed a W-shape-peak explicit solution as follows:

$$
u(\chi)=2-3 \cosh ^{2} \chi+(\cosh \chi+1 / 3) \sqrt{3(3 \cosh \chi+1)(\cosh \chi-1)},
$$

where $\chi=\left|x-\frac{11}{3} t\right| / 2-\ln 2$. 
Li and Zhang [3] called Eq. (1.1) a singular travelling wave equation. Following them, we call Eq. (1.1) a singular integrable equation. Using bifurcation method of dynamical systems, Li and Zhang [3] showed that there exist smooth solitary solutions and periodic waves of Eq. (1.1) when some parameter conditions are satisfied. They explained why the so-called W-shape-peaks and M-shape-peaks solutions can be created, gave the determined parameter conditions and got exact parametric expressions for all solitary wave solutions of Eq. (1.1). But they did not obtain exact parametric expressions of the smooth periodic waves.

In this paper, we employ the method of dynamical systems $[3,6,7,8]$ to investigate the periodic waves of the Eq. (1.1). Firstly, we derive travelling wave equation and system. Then we draw bifurcation curves and bifurcation phase portraits of the travelling wave system. By using these closed orbits, the exact periodic wave solutions of Eq. (1.1) are obtained. Corresponding to the special closed orbits the periodic waves have special loop cusp shape. We call them periodic cusp loop waves. The limit of the periodic cusp loop waves are cusp loop solitary waves. Our method is similar to the one used in [3]. Comparing our results with Qiao $[4,5]$ and Li et al. [3], we note that the periodic cusp loop waves and cusp loop solitary waves are new.

In order to state our main results in a compact form, for a given constant $c>0$ and $g \neq 0$, let the $\varphi_{1}, \varphi_{2}$ and $\varphi_{3}$ are three simple real zeros of $f(\varphi)=\varphi^{3}-$ $c \varphi+g, \varphi_{\sqrt{c}}\left(-\sqrt{c}<\varphi_{\sqrt{c}}<\sqrt{c}\right)$ is a simple real root of $\left(c-\varphi^{2}\right)^{2}+4 g \varphi=4 g \sqrt{c}$, $\varphi_{-\sqrt{c}}\left(-\sqrt{c}<\varphi_{-\sqrt{c}}<\sqrt{c}\right)$ is a simple real root of $\left(c-\varphi^{2}\right)^{2}+4 g \varphi=-4 g \sqrt{c}$, and the $\varphi_{0}$ is original value.

Proposition 1. (1) If $\frac{8 c \sqrt{c}}{27}<g<\frac{2 c}{3} \sqrt{\frac{c}{3}}$ and $\varphi_{2}<\varphi_{0}<\varphi_{3}$, then the Eq. (1.1) has a smooth periodic wave, and the smooth periodic wave becomes a smooth solitary wave when $\varphi_{0}$ tends to $\varphi_{2}$.

(2) If $g=\frac{8 c \sqrt{c}}{27}$ and $\varphi_{2}<\varphi_{0}<\varphi_{3}$, then the Eq. (1.1) has a smooth periodic wave, and the smooth periodic wave becomes a peakon wave when $\varphi_{0}$ tends to $\varphi_{2}$.

(3) If $0<g<\frac{8 c \sqrt{c}}{27}$ and $\varphi_{\sqrt{c}}<\varphi_{0}<\varphi_{3}$, then the Eq. (1.1) has a smooth periodic wave.

(4) If $0<g<\frac{8 c \sqrt{c}}{27}$ and $\varphi_{0}=\varphi_{\sqrt{c}}$, then the Eq. (1.1) has a periodic cusp wave.

(5) If $0<g<\frac{8 c \sqrt{c}}{27}$ and $\varphi_{2}<\varphi_{0}<\varphi_{\sqrt{c}}$, then the Eq. (1.1) has a periodic cusp loop wave, and it becomes a cusp loop solitary wave when $\varphi_{0}$ tends to $\varphi_{2}$.

Under one of the above conditions, the periodic solution $u(x, t)=\varphi(\xi)$ of the Eq. (1.1) has parametric type as follows:

$$
\left\{\begin{array}{l}
\varphi=\frac{1}{4 g}\left(h_{0}-\left(\frac{z_{2}-z_{1} n_{1}^{2} \operatorname{sn}^{2}\left(w, k_{1}\right)}{1-n_{1}^{2} \operatorname{sn}^{2}\left(w, k_{1}\right)}\right)^{2}\right), \\
\xi=\frac{4}{\sqrt{\left(z_{4}-z_{2}\right)\left(z_{3}-z_{1}\right)}}\left(z_{1} w+\left(z_{2}-z_{1}\right) \Pi\left(\arcsin \left(\operatorname{sn}\left(w, k_{1}\right)\right), n_{1}^{2}, k_{1}\right)\right),
\end{array}\right.
$$

where $h_{0}=\left(c-\varphi_{0}^{2}\right)^{2}+4 g \varphi_{0}$, the $z_{1}, z_{2}, z_{3}$ and $z_{4}$ are four real simple real zeros of $G(z)=\frac{1}{16 g^{2}}\left(z^{4}-2 h_{0} z^{2}+16 g^{2} z+h_{0}^{2}-16 g^{2} c\right), w=\frac{\sqrt{\left(z_{4}-z_{2}\right)\left(z_{3}-z_{1}\right)}}{4} v$ is a 
parameter variable, $k_{1}=\sqrt{\frac{\left(z_{3}-z_{2}\right)\left(z_{4}-z_{1}\right)}{\left(z_{4}-z_{2}\right)\left(z_{3}-z_{1}\right)}}$ is the modulus of Jacobian elliptic function and $n_{1}=\sqrt{\frac{z_{3}-z_{2}}{z_{3}-z_{1}}}$.

Proposition 2. (6) If $-\frac{2 c}{3} \sqrt{\frac{c}{3}}<g<-\frac{8 c \sqrt{c}}{27}$ and $\varphi_{1}<\varphi_{0}<\varphi_{2}$, then the $E q$. (1.1) has a smooth periodic wave, and the smooth periodic wave becomes a smooth solitary wave when $\varphi_{0}$ tends to $\varphi_{2}$.

(7) If $g=-\frac{8 c \sqrt{c}}{27}$ and $\varphi_{1}<\varphi_{0}<\varphi_{2}$, then the Eq. (1.1) has a smooth periodic wave, and the smooth periodic wave becomes a peakon wave when $\varphi_{0}$ tends to $\varphi_{2}$.

(8) If $-\frac{8 c \sqrt{c}}{27}<g<0$ and $\varphi_{1}<\varphi_{0}<\varphi_{-\sqrt{c}}$, then the Eq. (1.1) has a smooth periodic wave.

(9) If $-\frac{8 c \sqrt{c}}{27}<g<0$ and $\varphi_{0}=\varphi_{-\sqrt{c}}$, then the Eq. (1.1) has a periodic cusp wave.

(10) If $-\frac{8 c \sqrt{c}}{27}<g<0$ and $\varphi_{-\sqrt{c}}<\varphi_{0}<\varphi_{2}$, then the Eq. (1.1) has a periodic cusp loop wave, and it becomes a cusp loop solitary wave when $\varphi_{0}$ tends to $\varphi_{2}$.

Under one of the above conditions, the periodic solution $u(x, t)=\varphi(\xi)$ of the Eq. (1.1) has parametric type as follows:

$$
\left\{\begin{array}{l}
\varphi=\frac{1}{4 g}\left(h_{0}-\left(\frac{z_{3}-z_{4} n_{2}^{2} \mathrm{sn}^{2}\left(w, k_{2}\right)}{1-n_{2}^{2} \mathrm{sn}^{2}\left(w, k_{2}\right)}\right)^{2}\right) \\
\xi=\frac{4}{\sqrt{\left(z_{4}-z_{2}\right)\left(z_{3}-z_{1}\right)}}\left(z_{4} w+\left(z_{3}-z_{4}\right) \Pi\left(\arcsin \left(\operatorname{sn}\left(w, k_{2}\right)\right), n_{2}^{2}, k_{2}\right)\right),
\end{array}\right.
$$

where $h_{0}=\left(c-\varphi_{0}^{2}\right)^{2}+4 g \varphi_{0}$, the $z_{1}, z_{2}, z_{3}$ and $z_{4}$ are four real simple real zeros of $G(z)=\frac{1}{16 g^{2}}\left(z^{4}-2 h_{0} z^{2}-16 g^{2} z+h_{0}^{2}-16 g^{2} c\right), w=\frac{\sqrt{\left(z_{4}-z_{2}\right)\left(z_{3}-z_{1}\right)}}{4} v$ is a parameter variable, $k_{2}=\sqrt{\frac{\left(z_{3}-z_{2}\right)\left(z_{4}-z_{1}\right)}{\left(z_{4}-z_{2}\right)\left(z_{3}-z_{1}\right)}}$ is the modulus of Jacobian elliptic function and $n_{2}=\sqrt{\frac{z_{3}-z_{2}}{z_{4}-z_{2}}}$.

Example 1. Letting $c=20$ and $g=20$, then $\varphi_{1} \doteq-4.906733293, \varphi_{2} \doteq$ $1.059459801 \varphi_{3} \doteq 3.847273492$ and $\varphi_{\sqrt{c}} \doteq 3.063233706$. Taking $\varphi_{0}=3.5$, we have $h_{0}=340.0625, z_{1} \doteq-30.10556009, z_{2} \doteq 2.693759228, z_{3} \doteq 7.749999997$ and $z_{4} \doteq 19.66180086$. Substituting these data into (1.3), on $\xi-u$ plane we draw smooth periodic wave graph as Fig. 1 (a).

Taking $\varphi_{0}=\varphi_{\sqrt{c}}$, we have $h_{0} \doteq 357.7708764, z_{1} \doteq-30.42816464, z_{2}=0$, $z_{3} \doteq 10.61659926$ and $z_{4} \doteq 19.81156537$. Substituting these data into (1.3), on $\xi-u$ plane we draw periodic cusp wave graph as Fig. 1 (b).

Taking $\varphi_{0}=1.5$, we have $h_{0}=435.0625, z_{1} \doteq-31.79311168, z_{2} \doteq$ $-5.543893946, z_{3}=17.75$ and $z_{4} \doteq 19.58700563$. Substituting these data into (1.3), on $\xi-u$ plane we draw periodic cusp loop wave graph as Fig. 1 (c).

Taking $\varphi_{0}=\varphi_{2}$, we have $h_{0} \doteq 441.1184867, z_{1} \doteq-31.89728257, z_{2} \doteq$ -5.857807291 and $z_{3}=z_{4} \doteq 18.87749737$. Substituting these data into (1.3), on $\xi-u$ plane we draw cusp loop solitary wave graph as Fig. 1 (d). 


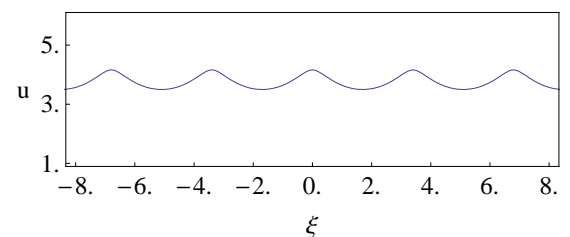

(a)

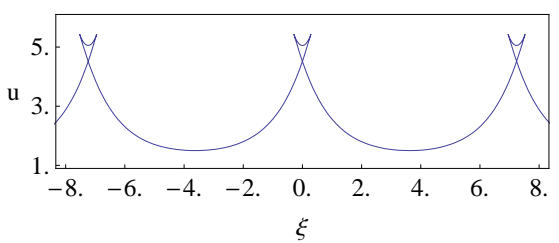

(c)

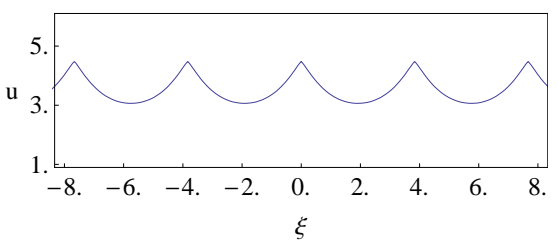

(b)

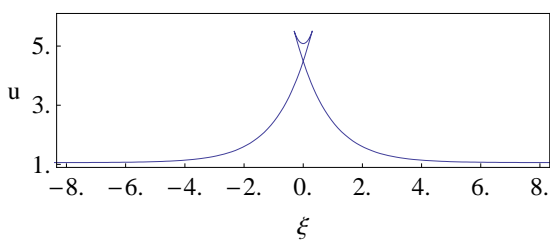

(d)

Figure 1. The smooth periodic wave, periodic cusp wave, periodic cusp loop wave and cusp loop solitary wave of Eq. (1.1) with $c=20$ and $g=20$ : (a) $\varphi_{0}=3.5$, (b) $\varphi_{0}=\varphi_{\sqrt{c}}$, (c) $\varphi_{0}=1.5$, (d) $\varphi_{0}=\varphi_{2}$.

Example 2. Letting $c=20$ and $g=-20$, then $\varphi_{1} \doteq-3.847273492, \varphi_{2} \doteq$ $-1.059459801 \varphi_{3} \doteq 4.906733293$ and $\varphi_{-\sqrt{c}} \doteq-3.063233706$. Taking $\varphi_{0}=$ -3.5, we have $h_{0}=340.0625, z_{1} \doteq-19.66180086, z_{2} \doteq-7.749999997, z_{3} \doteq$ -2.693759228 and $z_{4} \doteq 30.10556009$. Substituting these data into (1.4), on $\xi-u$ plane we draw smooth periodic wave graph as Fig. 2 (a).

Taking $\varphi_{0}=\varphi_{-\sqrt{c}}$, we have $h_{0}=357.7708764, z_{1} \doteq-19.81156537, z_{2} \doteq$ $-10.61659926, z_{3}=0$ and $z_{4} \doteq 30.42816464$. Substituting these data into (1.4), on $\xi-u$ plane we draw periodic cusp wave graph as Fig. 2 (b).

Taking $\varphi_{0}=-1.5$, we have $h_{0}=435.0625, z_{1} \doteq-19.58700563, z_{2}=$ $-17.75, z_{3} \doteq 5.543893946$ and $z_{4} \doteq 31.79311168$. Substituting these data into (1.4), on $\xi-u$ plane we draw periodic cusp loop wave graph as Fig. 2 (c).

Taking $\varphi_{0}=\varphi_{2}$, we have $h_{0} \doteq 441.1184867, z_{1}=z_{2} \doteq-18.87759249$, $z_{3} \doteq 5.857807291$ and $z_{4} \doteq 31.89728257$. Substituting these data into (1.4), on $\xi-u$ plane we draw cusp loop solitary wave graph as Fig. 2 (d).

\section{Preliminary}

In order to derive the expressions of the above solutions, we establish a planar system corresponding to Eq. (1.1). For a given constant $c$, substituting $\xi=$ $x-c t$ and $u(x, t)=\varphi(\xi)$ in Eq. (1.1), it follows that

$$
\left(\varphi^{2}-\left(\varphi^{\prime}\right)^{2}-c\right)\left(\varphi-\varphi^{\prime \prime}\right)^{\prime}+2 \varphi^{\prime}\left(\varphi-\varphi^{\prime \prime}\right)^{2}=0 .
$$

Integrating (2.1) once with respect to $\xi$, we have the following travelling wave equation.

$$
\left(\varphi-\varphi^{\prime \prime}\right)\left(\varphi^{2}-\left(\varphi^{\prime}\right)^{2}-c\right)+g=0
$$




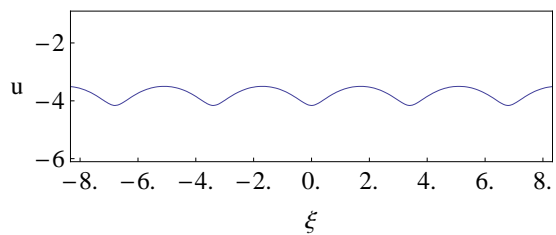

(a)

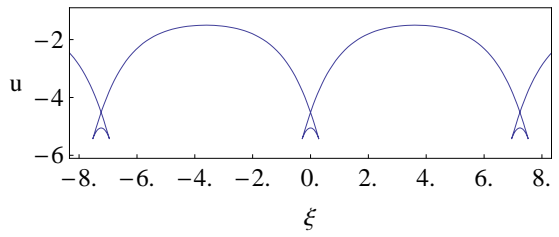

(c)

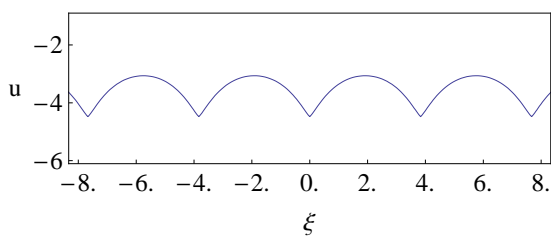

(b)

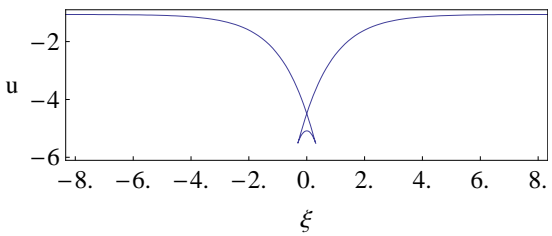

(d)

Figure 2. The smooth periodic wave, periodic cusp wave, periodic cusp loop wave and cusp loop solitary wave of Eq. (1.1) with $c=20$ and $g=-20$ : (a) $\varphi_{0}=-3.5$, (b) $\varphi_{0}=\varphi_{-\sqrt{c}}$, (c) $\varphi_{0}=-1.5$, (d) $\varphi_{0}=\varphi_{2}$.

where $g$ is integral constant. We suppose that $g \neq 0$, and letting $\varphi^{\prime}=y$, the Eq. (2.2) becomes a planar system

$$
\left\{\begin{array}{l}
\frac{d \varphi}{d \xi}=y, \\
\frac{d y}{d \xi}=\frac{\varphi\left(c-\varphi^{2}+y^{2}\right)-g}{c-\varphi^{2}+y^{2}} .
\end{array}\right.
$$

Clearly, on the hyperbola $\varphi^{2}-y^{2}=c$, the system (2.3) is discontinuous. Such system is called singular travelling wave system [3].

In what follows, without loss of generality we can assume $c>0$ and make the transformation

$$
d \xi=\left(c-\varphi^{2}+y^{2}\right) d \tau,
$$

where $\tau$ is parametric variable. Thus system (2.3) becomes

$$
\left\{\begin{array}{l}
\frac{d \varphi}{d \tau}=\left(c-\varphi^{2}+y^{2}\right) y \\
\frac{d y}{d \tau}=\varphi\left(c-\varphi^{2}+y^{2}\right)-g .
\end{array}\right.
$$

Obviously, systems (2.3) and (2.4) have the same first integral

$$
H(\varphi, y)=\left(c-\varphi^{2}+y^{2}\right)^{2}+4 g \varphi=h .
$$

Therefore, both systems (2.3) and (2.4) have the same topological phase portraits except the hyperbola $\varphi^{2}-y^{2}=c$.

Let $f_{0}(\varphi)=\varphi^{3}-c \varphi$, then for a fixed $c>0$, the $\varphi= \pm \sqrt{\frac{c}{3}}$ are extreme points of $f_{0}(\varphi), f_{0}\left(-\sqrt{\frac{c}{3}}\right)=\frac{2 c}{3} \sqrt{\frac{c}{3}}$ is maximum, and $f_{0}\left(\sqrt{\frac{c}{3}}\right)=-\frac{2 c}{3} \sqrt{\frac{c}{3}}$ is minimum. 
Let $f(\varphi)=\varphi^{3}-c \varphi+g$, then the following facts hold.

(1) $f(\varphi)$ only has a zero $\varphi_{1}<-\sqrt{c}$ when $g>\frac{2 c}{3} \sqrt{\frac{c}{3}} \cdot\left(\varphi_{1}, 0\right)$ is a saddle point of system (2.4).

(2) $f(\varphi)$ has one simple zero $\varphi_{1}$, a double zero $\varphi_{2}=\sqrt{\frac{c}{3}}$, and $\varphi_{1}<-\sqrt{c}<$ $\varphi_{2}$ when $g=\frac{2 c}{3} \sqrt{\frac{c}{3}}$. $\left(\varphi_{1}, 0\right)$ is a saddle point, and $\left(\varphi_{2}, 0\right)$ is a cusp point of system (2.4).

(3) $f(\varphi)$ has three simple zeros $\varphi_{1}, \varphi_{2}$ and $\varphi_{3}$, and $\varphi_{1}<-\sqrt{c}<0<\varphi_{2}<$ $\sqrt{\frac{c}{3}}<\varphi_{3}<\sqrt{c}$ when $0<g<\frac{2 c}{3} \sqrt{\frac{c}{3}} .\left(\varphi_{1}, 0\right)$ and $\left(\varphi_{2}, 0\right)$ are two saddle points, and $\left(\varphi_{3}, 0\right)$ is a center point of system $(2.4)$.

(4) $f(\varphi)$ has three simple zeros $\varphi_{1}, \varphi_{2}$ and $\varphi_{3}$, and $-\sqrt{c}<\varphi_{1}<-\sqrt{\frac{c}{3}}<$ $\varphi_{2}<0<\sqrt{c}<\varphi_{3}$ when $-\frac{2 c}{3} \sqrt{\frac{c}{3}}<g<0$. $\left(\varphi_{2}, 0\right)$ and $\left(\varphi_{3}, 0\right)$ are two saddle points, and $\left(\varphi_{1}, 0\right)$ is a center point of system $(2.4)$.

(5) $f(\varphi)$ has one simple zero $\varphi_{2}$, a double zero $\varphi_{1}=-\sqrt{\frac{c}{3}}$, and $\varphi_{1}<\sqrt{c}<$ $\varphi_{2}$ when $g=-\frac{2 c}{3} \sqrt{\frac{c}{3}}$. $\left(\varphi_{1}, 0\right)$ is a cusp point, and $\left(\varphi_{2}, 0\right)$ is a saddle point of system $(2.4)$.

(6) $f(\varphi)$ only has a zero $\varphi_{1}>\sqrt{c}$ when $g<-\frac{2 c}{3} \sqrt{\frac{c}{3}} \cdot\left(\varphi_{1}, 0\right)$ is a saddle point of system $(2.4)$.

When $|g|<\frac{2 c}{3} \sqrt{\frac{c}{3}}$ and $g \neq 0$, the homoclinic orbit is defined by $H(\varphi, y)=$ $H\left(\varphi_{2}, 0\right)=h_{2}$. The homoclinic orbit that passes the point $(\sqrt{c}, 0)($ or $(-\sqrt{c}, 0))$ is defined by $g=\frac{8 c \sqrt{c}}{27}$ (or $g=-\frac{8 c \sqrt{c}}{27}$ ).

According to the above analysis, we obtain the bifurcation phase portraits of systems (2.3) and (2.4) as given in Fig. 3.

\section{The Proof of Main Results}

It is well known that the closed orbit of the travelling system gives a periodic wave solution of the corresponding nonlinear wave equation. To find the exact parametric expressions of periodic wave solutions, we assume that $\left(\varphi_{0}, 0\right)$ is the initial point of a closed orbit, and it has expression from (2.5) that

$$
y^{2}=\varphi^{2}+\delta \sqrt{h_{0}-4 g \varphi}-c
$$

where $\delta= \pm 1$ and $h_{0}=H\left(\varphi_{0}, 0\right)$. Let $z^{2}=h_{0}-4 g \varphi$, we have

$$
\varphi=\frac{h_{0}-z^{2}}{4 g}, \quad y^{2}=G(z),
$$

where $G(z)=\frac{1}{16 g^{2}}\left(z^{4}-2 h_{0} z^{2}+\delta 16 g^{2} z+h_{0}^{2}-16 g^{2} c\right)$. From first equation of (2.3), we obtain $\frac{d z}{d \xi}=-\frac{2 g}{z} y$. Let

$$
d \xi=z d v
$$

then

$$
\frac{d z}{d v}=-2 g y
$$




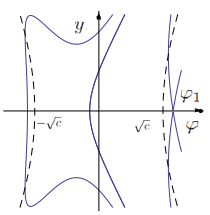

(a)

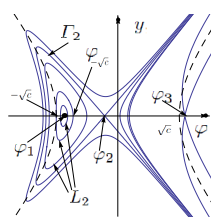

(e)

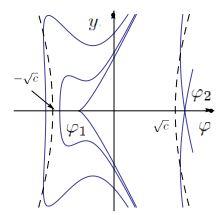

(b)

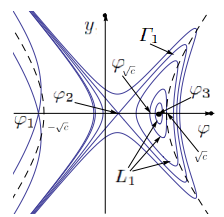

(f)

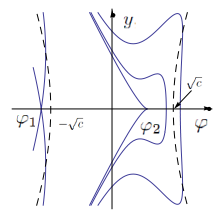

(i)

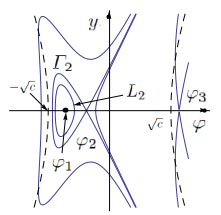

(c)

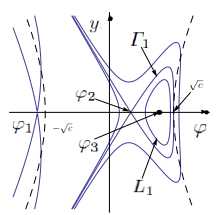

(g)

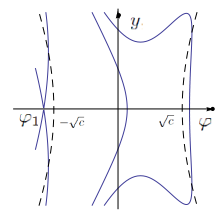

(j)

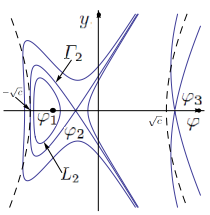

(d)

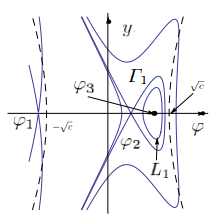

(h)

Figure 3. The bifurcation phase portraits of systems (2.3) and (2.4) with $c>0$ and $g \neq 0$ : (a) $g<-\frac{2 c}{3} \sqrt{\frac{c}{3}} ;$ (b) $g=-\frac{2 c}{3} \sqrt{\frac{c}{3}} ;$ (c) $-\frac{2 c}{3} \sqrt{\frac{c}{3}}<g<-\frac{8 c \sqrt{c}}{27}$; (d) $g=-\frac{8 c \sqrt{c}}{27}$; (e) $-\frac{8 c \sqrt{c}}{27}<g<0$; (f) $0<g<\frac{8 c \sqrt{c}}{27}$; (g) $g=\frac{8 c \sqrt{c}}{27}$; (h) $\frac{8 c \sqrt{c}}{27}<g<\frac{2 c}{3} \sqrt{\frac{c}{3}}$; (i) $g=\frac{2 c}{3} \sqrt{\frac{c}{3}}$; (j) $g>\frac{2 c}{3} \sqrt{\frac{c}{3}}$.

\subsection{The proof of Proposition 1}

(1) $\frac{8 c \sqrt{c}}{27}<g<\frac{2 c}{3} \sqrt{\frac{c}{3}}$ and $\varphi_{2}<\varphi_{0}<\varphi_{3}$. In this case, the closed orbit $L_{1}$ which passes through the point $\left(\varphi_{0}, 0\right)$ and the homoclinic orbit $\Gamma_{1}$ which passes through the point $\left(\varphi_{2}, 0\right)$ have no intersection point with the hyperbola $\varphi^{2}-y^{2}=c$ (see Fig. $\left.3(\mathrm{~h})\right)$. Thus, corresponding to $L_{1}$, the Eq. (1.1) has a smooth periodic wave, and corresponding to $\Gamma_{1}$, the Eq. (1.1) has a smooth solitary wave. The smooth periodic wave becomes a smooth solitary wave when $\varphi_{0}$ tends to $\varphi_{2}$.

(2) $g=\frac{8 c \sqrt{c}}{27}$ and $\varphi_{2}<\varphi_{0}<\varphi_{3}$. In this case, the closed orbit $L_{1}$ which passes through the point $\left(\varphi_{0}, 0\right)$ has no intersection point with the hyperbola $\varphi^{2}-y^{2}=c$, and The homoclinic orbit $\Gamma_{1}$ which passes through the point $\left(\varphi_{2}, 0\right)$ has only one intersection point $(\sqrt{c}, 0)$ with the hyperbola $\varphi^{2}-y^{2}=c$ (see Fig. 3 (g)). Thus, corresponding to $L_{1}$, the Eq. (1.1) has a smooth periodic wave, and corresponding to $\Gamma_{1}$, the Eq. (1.1) has a peakon. The smooth periodic wave becomes a peakon when $\varphi_{0}$ tends to $\varphi_{2}$.

(3) $0<g<\frac{8 c \sqrt{c}}{27}$ and $\varphi_{\sqrt{c}}<\varphi_{0}<\varphi_{3}$, where $H\left(\varphi_{\sqrt{c}}, 0\right)=H(\sqrt{c}, 0)$. In this case, the closed orbit $L_{1}$ which passes through the point $\left(\varphi_{0}, 0\right)$ has no intersection point with the hyperbola $\varphi^{2}-y^{2}=c$ (see Fig. 3 (f)). Thus, 
corresponding to $L_{1}$, the Eq. (1.1) has a smooth periodic wave.

(4) $0<g<\frac{8 c \sqrt{c}}{27}$ and $\varphi_{0}=\varphi_{\sqrt{c}}$. In this case, the closed orbit $L_{1}$ which passes through the point $\left(\varphi_{0}, 0\right)$ has only a intersection point $(\sqrt{c}, 0)$ with the hyperbola $\varphi^{2}-y^{2}=c$ (see Fig. 3 (f)). Thus, corresponding to $L_{1}$, the Eq. (1.1) has a periodic cusp wave.

(5) $0<g<\frac{8 c \sqrt{c}}{27}$ and $\varphi_{2}<\varphi_{0}<\varphi_{\sqrt{c}}$.

In this case, the closed orbit $L_{1}$ which passes through the point $\left(\varphi_{0}, 0\right)$ and the homoclinic orbit $\Gamma_{1}$ which passes through the point $\left(\varphi_{2}, 0\right)$ have two intersection points with the hyperbola $\varphi^{2}-y^{2}=c$ (see Fig. 3 (f)). Thus, corresponding to $L_{1}$, the Eq. (1.1) has a periodic cusp loop wave, and corresponding to $\Gamma_{1}$, the Eq. (1.1) has a cusp loop solitary wave. The periodic cusp loop wave becomes cusp loop solitary wave when $\varphi_{0}$ tends to $\varphi_{2}$.

Under the one of conditions (1), (2), (3), (4) and (5), the closed orbit $L_{1}$ has expression as $(3.1)$, where $\delta=1$, and $G(z)=\frac{1}{16 g^{2}}\left(z^{4}-2 h z^{2}+16 g^{2} z+\right.$ $\left.h^{2}-16 g^{2} c\right)=\frac{1}{16 g^{2}}\left(z-z_{1}\right)\left(z-z_{2}\right)\left(z-z_{3}\right)\left(z-z_{4}\right), z_{1}, z_{2}, z_{3}$ and $z_{4}$ are four real roots of the $G(z)=0$. Thus, the second equation of (3.2) becomes

$$
y= \pm \frac{1}{4 g} \sqrt{\left(z_{4}-z\right)\left(z_{3}-z\right)\left(z-z_{2}\right)\left(z-z_{1}\right)},
$$

where $z_{1}<z_{2} \leq z \leq z_{3}<z_{4}$.

Substituting (3.5) into (3.4) and integrating it along $L_{1}$, we have

$$
\int_{z_{2}}^{z} \frac{1}{\sqrt{\left(z_{4}-s\right)\left(z_{3}-s\right)\left(s-z_{2}\right)\left(s-z_{1}\right)}} d s=\mp \frac{1}{2} \int_{0}^{v} d s .
$$

By using formula 254.00 in [1], from (3.6), we obtain

$$
z=\frac{z_{2}-z_{1} n_{1}^{2} \operatorname{sn}^{2}\left(w, k_{1}\right)}{1-n_{1}^{2} \operatorname{sn}^{2}\left(w, k_{1}\right)}
$$

where $w=\frac{\sqrt{\left(z_{4}-z_{2}\right)\left(z_{3}-z_{1}\right)}}{4} v$ is a parameter variable, $k_{1}=\sqrt{\frac{\left(z_{3}-z_{2}\right)\left(z_{4}-z_{1}\right)}{\left(z_{4}-z_{2}\right)\left(z_{3}-z_{1}\right)}}$ is the modulus of Jacobian elliptic function and $n_{1}=\sqrt{\frac{z_{3}-z_{2}}{z_{3}-z_{1}}}$.

Substituting (3.7) into (3.3) and integrating it, we have

$$
\int_{0}^{\xi} d s=\frac{4}{\sqrt{\left(z_{4}-z_{2}\right)\left(z_{3}-z_{1}\right)}} \int_{0}^{w} \frac{z_{2}-z_{1} n_{1}^{2} \operatorname{sn}^{2}\left(s, k_{1}\right)}{1-n_{1}^{2} \operatorname{sn}^{2}\left(s, k_{1}\right)} d s .
$$

By using formula 400.01 in [1], from (3.8), we obtain

$$
\xi=\frac{4}{\sqrt{\left(z_{4}-z_{2}\right)\left(z_{3}-z_{1}\right)}}\left(z_{1} w+\left(z_{2}-z_{1}\right) \Pi\left(\arcsin \left(\operatorname{sn}\left(w, k_{1}\right)\right), n_{1}^{2}, k_{1}\right)\right) \text {. }
$$

Thus, from (3.2) we obtain the periodic wave solution $u(x, t)=\varphi(\xi)$ of parametric type as follows:

$$
\left\{\begin{array}{l}
\varphi=\frac{1}{4 g}\left(h_{0}-z^{2}\right), \\
\xi=\frac{4}{\sqrt{\left(z_{4}-z_{2}\right)\left(z_{3}-z_{1}\right)}}\left(z_{1} w+\left(z_{2}-z_{1}\right) \Pi\left(\arcsin \left(\operatorname{sn}\left(w, k_{1}\right)\right), n_{1}^{2}, k_{1}\right)\right),
\end{array}\right.
$$

Here we complete the proof of Proposition 1. 


\subsection{The proof of Proposition 2}

(6) $-\frac{2 c}{3} \sqrt{\frac{c}{3}}<g<-\frac{8 c \sqrt{c}}{27}$ and $\varphi_{1}<\varphi_{0}<\varphi_{2}$. In this case, the closed orbit $L_{2}$ which passes through the point $\left(\varphi_{0}, 0\right)$ and the homoclinic orbit $\Gamma_{2}$ which passes through the point $\left(\varphi_{2}, 0\right)$ have no intersection point with the hyperbola $\varphi^{2}-y^{2}=c$ (see Fig. 3 (c)). Thus, corresponding to $L_{2}$, the Eq. (1.1) has a smooth periodic wave, and corresponding to $\Gamma_{2}$, the Eq. (1.1) has a smooth solitary wave. The smooth periodic wave becomes a smooth solitary wave when $\varphi_{0}$ tends to $\varphi_{2}$.

(7) $g=-\frac{8 c \sqrt{c}}{27}$ and $\varphi_{1}<\varphi_{0}<\varphi_{2}$. In this case, the closed orbit $L_{2}$ which passes through the point $\left(\varphi_{0}, 0\right)$ has no intersection point with the hyperbola $\varphi^{2}-y^{2}=c$, and the homoclinic orbit $\Gamma_{2}$ which passes through the point $\left(\varphi_{2}, 0\right)$ has only one intersection point $(-\sqrt{c}, 0)$ with the hyperbola $\varphi^{2}-y^{2}=c$ (see Fig. $3(\mathrm{~d}))$. Thus, corresponding to $L_{2}$, the Eq. (1.1) has a smooth periodic wave, and corresponding to $\Gamma_{2}$, the Eq. (1.1) has a peakon. The smooth periodic wave becomes a peakon when $\varphi_{0}$ tends to $\varphi_{2}$.

(8) $-\frac{8 c \sqrt{c}}{27}<g<0$ and $\varphi_{1}<\varphi_{0}<\varphi_{-\sqrt{c}}$, where $H\left(\varphi_{-\sqrt{c}}, 0\right)=H(-\sqrt{c}, 0)$.

In this case, the closed orbit $L_{2}$ which passes through the point $\left(\varphi_{0}, 0\right)$ has no intersection point with the hyperbola $\varphi^{2}-y^{2}=c$ (see Fig. 3 (e)). Thus, corresponding to $L_{2}$, the Eq. (1.1) has a smooth periodic wave.

(9) $-\frac{8 c \sqrt{c}}{27}<g<0$ and $\varphi_{0}=\varphi_{-\sqrt{c}}$.

In this case, the closed orbit $L_{2}$ which passes through the point $\left(\varphi_{0}, 0\right)$ has only a intersection point $(-\sqrt{c}, 0)$ with the hyperbola $\varphi^{2}-y^{2}=c$ (see Fig. 3 (e)). Thus, corresponding to $L_{2}$, the Eq. (1.1) has a periodic cusp wave.

(10) $-\frac{8 c \sqrt{c}}{27}<g<0$ and $\varphi_{-\sqrt{c}}<\varphi_{0}<\varphi_{2}$.

In this case, the closed orbit $L_{2}$ which passes through the point $\left(\varphi_{0}, 0\right)$ and the homoclinic orbit $\Gamma_{2}$ which passes through the point $\left(\varphi_{2}, 0\right)$ have two intersection points with the hyperbola $\varphi^{2}-y^{2}=c$ (see Fig. $3(\mathrm{e})$ ). Thus, corresponding to $L_{2}$, the Eq. (1.1) has a periodic cusp loop wave, and corresponding to $\Gamma_{2}$, the Eq. (1.1) has a cusp loop solitary wave. The periodic cusp loop wave becomes cusp loop solitary wave when $\varphi_{0}$ tends to $\varphi_{2}$.

Under the one of conditions (6), (7), (8), (9) and (10), the closed orbit $L_{2}$ has expression as (3.1), where $\delta=-1$, and $G(z)=\frac{1}{16 g^{2}}\left(z^{4}-2 h z^{2}-16 g^{2} z+\right.$ $\left.h^{2}-16 g^{2} c\right)=\frac{1}{16 g^{2}}\left(z-z_{1}\right)\left(z-z_{2}\right)\left(z-z_{3}\right)\left(z-z_{4}\right), z_{1}, z_{2}, z_{3}$ and $z_{4}$ are four real roots of the $G(z)=0$. Thus, the second equation of (3.2) becomes

$$
y= \pm \frac{1}{4|g|} \sqrt{\left(z_{4}-z\right)\left(z_{3}-z\right)\left(z-z_{2}\right)\left(z-z_{1}\right)},
$$

where $z_{1}<z_{2} \leq z \leq z_{3}<z_{4}$.

Substituting (3.11) into (3.4) and integrating it along $L_{2}$, we have

$$
\int_{z}^{z_{3}} \frac{1}{\sqrt{\left(z_{4}-s\right)\left(z_{3}-s\right)\left(s-z_{2}\right)\left(s-z_{1}\right)}} d s= \pm \frac{1}{2} \int_{v}^{0} d s .
$$

By using formula 255.00 in [1], from (3.12), we obtain

$$
z=\frac{z_{3}-z_{4} n_{2}^{2} \operatorname{sn}^{2}\left(w, k_{2}\right)}{1-n_{2}^{2} \operatorname{sn}^{2}\left(w, k_{2}\right)}
$$


where $w=\frac{\sqrt{\left(z_{4}-z_{2}\right)\left(z_{3}-z_{1}\right)}}{4} v$ is a parameter variable, $k_{2}=\sqrt{\frac{\left(z_{3}-z_{2}\right)\left(z_{4}-z_{1}\right)}{\left(z_{4}-z_{2}\right)\left(z_{3}-z_{1}\right)}}$ is the modulus of Jacobian elliptic function and $n_{2}=\sqrt{\frac{z_{3}-z_{2}}{z_{4}-z_{2}}}$.

Substituting (3.13) into (3.3) and integrating it, we have

$$
\int_{0}^{\xi} d s=\frac{4}{\sqrt{\left(z_{4}-z_{2}\right)\left(z_{3}-z_{1}\right)}} \int_{0}^{w} \frac{z_{3}-z_{4} n_{2}^{2} \operatorname{sn}^{2}\left(s, k_{2}\right)}{1-n_{2}^{2} \operatorname{sn}^{2}\left(s, k_{2}\right)} d s .
$$

By using formula 400.01 in [1], from (3.14), we obtain

$$
\xi=\frac{4}{\sqrt{\left(z_{4}-z_{2}\right)\left(z_{3}-z_{1}\right)}}\left(z_{4} w+\left(z_{3}-z_{4}\right) \Pi\left(\arcsin \left(\operatorname{sn}\left(w, k_{2}\right)\right), n_{2}^{2}, k_{2}\right)\right) \text {. }
$$

Thus, from (3.2) we obtain the periodic wave solution $u(x, t)=\varphi(\xi)$ of parametric type as follows:

$$
\left\{\begin{array}{l}
\varphi=\frac{1}{4 g}\left(h_{0}-z^{2}\right), \\
\xi=\frac{4}{\sqrt{\left(z_{4}-z_{2}\right)\left(z_{3}-z_{1}\right)}}\left(z_{4} w+\left(z_{3}-z_{4}\right) \Pi\left(\arcsin \left(\operatorname{sn}\left(w, k_{2}\right)\right), n_{2}^{2}, k_{2}\right)\right),
\end{array}\right.
$$

Here we complete the proof of Proposition 2.

\section{Conclusions}

In this paper, we obtained exact periodic wave solutions of Eq. (1.1) by using the theory of dynamical systems. We draw the bifurcation phase portraits of the singular travelling wave system (2.3). Through studying shape of periodic waves, we have shown that the periodic waves of Eq. (1.1) contain smooth periodic waves, periodic cusp waves and periodic cusp loop waves. The limit of periodic cusp loop waves are cusp loop solitary waves (see Figs. 1 and 2). Compare the results with Qiao [4, 5] and Li et al. [3], the periodic cusp loop waves and cusp loop solitary waves are new. The Eq. (1.1) naturally has a physical meaning since it is derived from the two dimensional Euler equation (see [4]). In this paper, we successfully solve the Eq. (1.1) with smooth periodic waves, periodic cusp waves, periodic cusp loop waves and cusp loop solitary waves. The solutions of Eq. (1.1) may be applied to neuroscience for providing a mathematical model and explaining electrophysiological responses of visceral nociceptive neurons and sensitization of dorsal root reflexes [2].

\section{Acknowledgements}

The authors express their sincere thanks to the reviewer for suggestions and comments. This work was supported by the Natural Science Foundation of China (No. 10871073) and Yunnan Province (2008ZC153M). 


\section{References}

[1] P.F. Byrd and M.D. Friedman. Handbook of Elliptic Integrals for Engineers and Scientists. Springer, Berlin, 1971.

[2] J.H. Chen, H.R. Weng and P.M. Dougherty. Sensitization of dorsal root reflexes in vitro and hyperalgesia in neonatal rats produced by capsaicin. Neuroscience, 126:734-751, 2004. Doi:10.1016/j.neuroscience.2004.04.021.

[3] J.B. Li and Y.Zhang. Exact M/W-shape solitary wave solutions determined by a singular traveling wave equation. Nonlinear Analysis: Real World Applications, 10:1797-1802, 2009. Doi:10.1016/j.nonrwa.2008.02.016.

[4] Z.J. Qiao. A new integrable equation with cuspons and W/M-shape peaks solitons. J. Math. Phys., 47:112701: 1-9, 2006. Doi:10.1063/1.2365758.

[5] Z.J. Qiao. New integrable hierarchy, its parametric solutions, cuspons, one-peak solitons, and M/W-shape peak solitons. J. Math. Phys., 48:082701: 1-20, 2007. Doi:10.1063/1.2759830.

[6] W.G. Rui, S.L. Xie, B. He and Y. Long. Integral bifurcation method and its application for solving the modified equal width wave equation and its variants. Rostock. Math. Kolloq., 62:87-106, 2007.

[7] S.L. Xie and J.H. Cai. Exact compacton and generalized kink wave solutions of the extended reduced Ostrovsky equation. Commun Nonlinear Sci. Numer. Simulat., 14:3561-3573, 2009. Doi:10.1016/j.cnsns.2009.02.001.

[8] S.L. Xie, W.G. Rui and X.C. Hong. The compactons and generalized kink waves to a generalized Camassa-Holm equation. Rostock. Math. Kolloq., 61:31-48, 2006. 